\title{
Umbrellas and Bathrooms
}

\section{Emiel Krahmer and Reinhard Muskens Tilburg University}

\section{Two problems for DRT, and a reduction}

\subsection{The double negation problem: umbrellas}

In a now classic paper (Karttunen [1976]) Karttunen noted that while a discourse referent cannot outlive a single negation or a single verb with an inherently negative implication (such as fail, neglect or forget) it will not be blocked by a double negation. While in (1) the pronoun it cannot be interpreted as dependent on $a$ question and in (2) the pronoun cannot depend on an answer, the definite in (3) may depend on the preceding indefinite and the it in (4) can be taken to refer to an umbrella. The anaphoric pronouns in (5) can likewise be interpreted as depending on the indefinite that preceeds them, even though the latter is within the scope of two negations. ${ }^{1}$

(1) Bill didn't dare to ask a question. *The lecturer answered it.

(2) John failed to find an answer. *It was wrong.

(3) John didn't fail to find an answer. The answer was even right.

(4) John didn't remember not to bring an umbrella, although we had no room for it.

(5) It is not true that John didn't bring an umbrella. It was purple and it stood in the hallway.

Various authors ${ }^{2}$ have pointed out that examples such as (3), (4) and (5) are a problem for dynamic theories of discourse such as Discourse Representation Theory (DRT, Kamp [1981], Kamp \& Reyle [1993]), File Change Semantics (FCS, Heim [1982, 1983]) or Dynamic Predicate Logic (DPL, Groenendijk \& Stokhof [1991]). These theories correctly predict negation to be a plug with respect to anaphoric binding and thus fit the facts in (1) and (2), 3 but they also incorrectly predict a double negation to be a double plug, not a plug unplugged as the facts in (3)-(5) would suggest. In DRT for example, the discourse referent that is connected to an umbrella in the first sentence of (5) will land up in a Discourse Representation Structure (DRS, or box henceforth) that is twice embedded to the main DRS and that will thus not be accessible for future anaphoric reference. An application of the DRT construction algorithm to the first sentence of (5) gives the box in (6) as an output, while it is the simpler box in (7) that would give the right predictions here. In the latter, but not in the former, the discourse referent $y$, which is connected to an umbrella, will be accessible from conditions in the main DRS. 
(6)

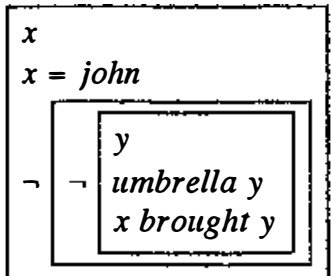

(7)

$$
\begin{array}{|l|}
x \quad y \\
x=\text { john } \\
\text { umbrella } y \\
x \text { brought } y
\end{array}
$$

Other formulations of the dynamic perspective are confronted with essentially the same difficulty. In DPL, the negation of a formula $\varphi$ will act as a 'test', irrespective of the intemal structure of $\varphi$, and so, since the first clause of (5) is of the form $\neg \varphi$, the anaphoric link between an umbrella and it is predicted to be impossible. In FCS we have that the first sentence in (5) does not succeed in extending the domain of the current file, while a new card for an umbrella is needed in order to establish the link between antecedent and anaphoric pronoun. In this paper we shall discuss the double negation problem (and the disjunction problem-see below) from a DRT perspective, but the reader will have no difficulty in translating our proposed solutions to her favourite dynamic semantic framework.

While we think that Karttunen's data essentially show that double negations are holes for anaphoric linking and thus form a problem for the standard dynamic accounts of anaphora, it should be noted that other data apparently point in an opposite direction. Consider ( $8 \mathrm{a}$ ) for example, a text that is decidedly odd if the anaphoric pronoun is interpreted as depending on no guest, even though the latter occurs within the scope of a negation and no other relevant operators intervene between the would-be antecedent and its dependent element.

(8a) It is not true that there is no guest at this wedding. ??He is standing right behind you.

(8b) It is not true that there is no bride at this wedding. She is standing right behind you.

The oddity of (8a) should be contrasted however with the complete acceptability of (8b) and is due, we conjecture, to a uniqueness effect (cf. Evans [1977], Kadmon [1987]). Given some highly unlikely context in which it is understood between speaker and hearer that at most one guest can be present at a wedding (8a) would be fine. We feel that it is precisely the unlikelihood of such a context which explains the markedness of (8a). Note, however, that (8a) is still better than (9), its counterpart with one negation only. 
(9) There is no guest at this wedding. ${ }^{*} \mathrm{He}$ is standing right behind you.

Another category of prima facie counterexamples to the double negation rule is formed by cases where the only plugs intervening between a possible antecedent and an anaphoric element are indeed two negations, but where the two still do not conspire to form an authentic double negation because they sandwich other material. We have in mind cases like (10), whose first sentence should be rendered as the box in (11). Clearly this is as much a case of double negation as the sequence $\neg \exists x \neg$ is a case of double negation in predicate logic.

(10) No man didn't bring an umbrella. *It was purple and it stood in the hallway.

(11)

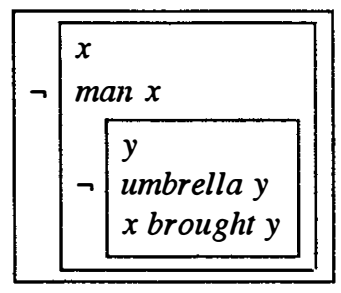

Since such apparent counterexamples on closer examination turn out to be no counterexamples at all, it seems we can take it as a general rule that as far as truth conditions and the possibility of anaphora are concerned double negations in standard English behave as if no negation at all were present.

\subsection{The disjunction problem: bathrooms}

The double negation problem seems to be related to another one that is also generally thought to be a hard nut for DRT and related theories. In (12) 4 the pronoun it is naturally linked to no bathroom, while DRT and other dynamic theories predict no antecedent in one part of a disjunction to be accessible for a pronoun in the other part. If we apply the standard construction rules to this sentence we get the DRS which is given in (13), but in this box the pronoun it cannot be resolved as the referent $x$.

(12) Either there's no bathroom in this house, or it's in a funny place

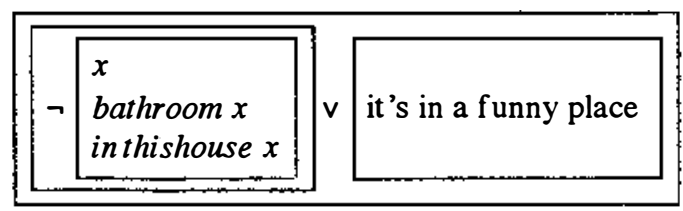

Kamp \& Reyle [1993 $]^{5}$ remark that it is in fact the presence of a negative element in the first disjunct which seems to license the anaphora in (12), even though 
negations in themselves usually block the possibility of linking. If there is no such negative element, as in (14), coref erence is impossible.

\section{?? Jones owns a car or he hides it}

A second observation made by Kamp \& Reyle is that sentences of the form $A$ or $B$ can in general be felicitously paraphrased as $A$ or otherwise $B$ and this leads to a proposal to let the DRT construction algorithm provide for the 'other case'. In (14) the 'other case' is the case where Jones does not own a car, and thus a revised form of the construction algorithm adds a condition to this effect to the second disjunct of the DRS for the sentence. The result is shown in (15).

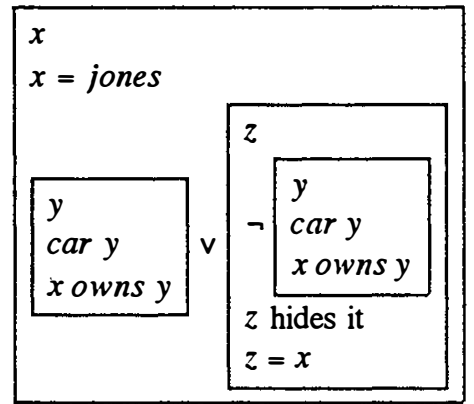

Here, since it cannot be resolved as $y$, the revised construction algorithm does not lead to predictions different from the original one, but as soon as we turn to sentences like (12) we see that Kamp \& Reyle's revision pays off. The 'other case' to be considered now is the case where a bathroom is present and if this information is added to that of the second dis junct we get (16) at a crucial stage of the DRS construction. This time it is possible to resolve it as $x$ and the link between anaphor and antecedent can be established.

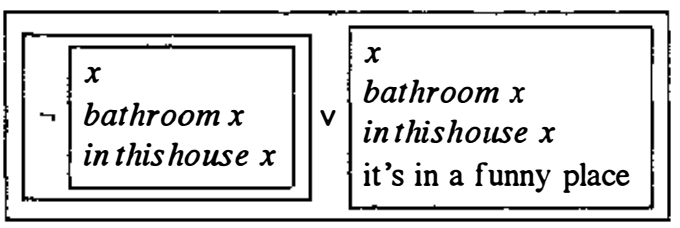

Kamp \& Reyle's treatment of 'bathroom' sentences can perhaps be criticised for not being entirely precise, in the sense that their new construction rule does not seem to prescribe exactly what material is to be added to the second disjunct. Suppose that we take the rule to be that in construing the DRS for a disjunction we should add the negation of the DRS for the first disjunct as a condition to the DRS for the second disjunct (call this Rule A). Then the DRS associated with (14) would indeed be (15), but the DRS for (12) would be (17) instead of (16), i.e. we get a double negation where we want no negation at all. 6 
(17)

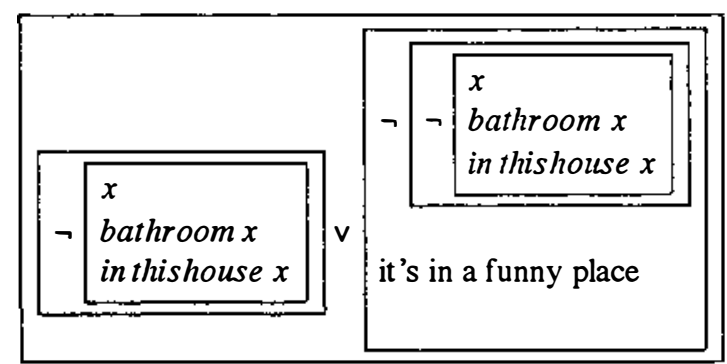

Note the structural similarity between the problem how to get from (17) to (16) and our previous problem how to obtain (7) from (6). In both cases we should like to be able to erase the double negation. An explicit rule to this effect would be very much ad hoc however and would be quite unlike all other DRT construction rules. It would have the useful property of being able to make certain referents accessible to certain pronouns (e.g. the referent $x$ is accessible from $i t$ in (16) but not in (17)) but this very property would also make it be theoretically suspicious for not being meaning preserving. If meanings determine context change potentials, as the dynamic perspective has it, then a rule to erase double negations that would change (6) into (7) (and (17) into (16)) cannot be meaning preserving since (6) gives a context which does not allow reference to $y$ while (7) gives one which does.

There is another difficulty with Kamp \& Reyle's proposed solution to the problem of 'bathroom' sentences: (16) simply does not have the truth conditions that (12) seems to have. Suppose there are in fact two toilets in the house, one of which is, and one of which is not in a strange place; then (12) is false according to our intuitions, but (16) is true since its second disjunct can be verified. We therefore turn to an earlier proposal from Roberts [1989], who renders (12) as (18). ${ }^{7}$ The idea here is that the material under the negation in the first disjunct is accomodated to provide an antecedent to the second disjunct. Since the first disjunct gives a negative answer to the question whether there is a toilet in the house, it is natural to interpret the second disjunct as pertaining to the possibility that there is one.

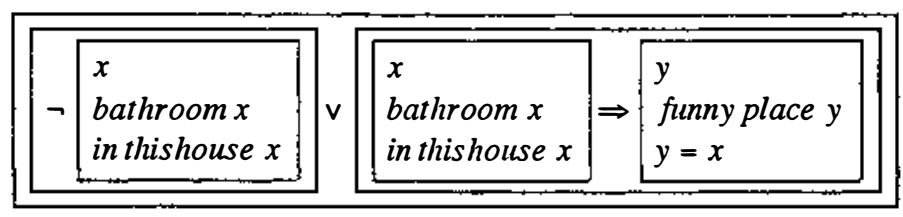

From a formal point of view it should be observed that, in a sense which will be made precise in the following section, (18) is equivalent to (19), the second disjunct of its only condition. And indeed, we feel that this is correct, since intuitively (12) is equivalent to (20). 
(19)

\begin{tabular}{l}
$\mid \begin{array}{l}x \\
\text { bathroom } x \\
\text { in thishouse } x\end{array}$ \\
\hline
\end{tabular}$\Rightarrow \begin{aligned} & y \\
& \text { funny place } y \\
& y=x\end{aligned}$

If there's a bathroom in this house it's in a funny place

How can we revise the DRT construction algorithm so that it gives (19) instead of (13) as an output for (12)? Here again we see that if we could but solve the double negation problem we would have a solution to the disjunction problem as well. For suppose that we would revise the construction algorithm so that at any time that a sentence disjunction $A$ or $B$ is encountered a condition of the form (21) (instead of the equivalent $A \vee B$ ) would be added to the current DRS (call this rule B); ${ }^{8}$ then (22) would be the output for (14), but for (12) DRS (23) would be obtained. The first of these is indeed correct in the sense that the anaphoric link is predicted to be impossible, but in the second we have a double negation again where no negation at all is wanted. The problem how to get from (23) to (19) is formally similar to the problem how to get from (17) to (16) or indeed to the question how to get (7) from (6). In this sense it can be said that the disjunction problem reduces to the double negation problem.

(21)

$$
-A \Rightarrow B
$$

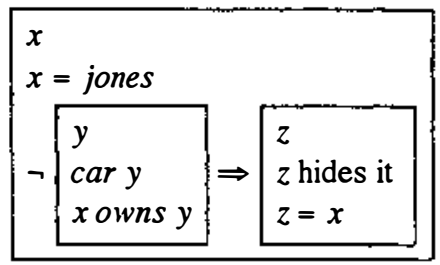

(23)

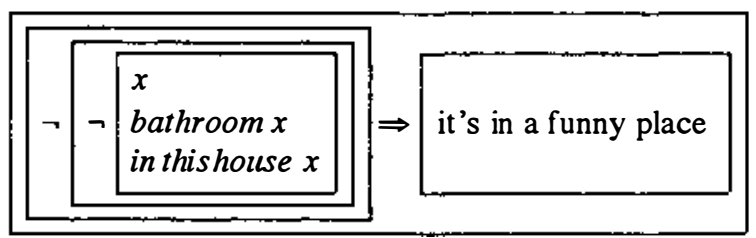

It thus seems that if we can revise the DRT box language by adding a new negation which obeys the law of double negations (i.e. which allows for cancelling double negations) we may not only solve the problems that we have encountered with Karttunen's 'umbrella' sentences, but we may also be able to deal with 'bathroom' sentences. An attempt to carry out such a revision will be made in section 3 below, but first let us look into the syntax and semantics of the standard box language in some detail. 


\section{Standard DRT: the formalities}

The Double Negation DRT of the next section will be a generalisation of standard DRT and for the sake of easy comparison we shall give concise versions of the most important DRT definitions in this one. In fact, we shall extend the standard syntax slightly and add a sequencing operator ';' which takes two boxes and gives a complex box. ${ }^{9}$ This addition, which seems natural in itself, in fact takes us already half way from the standard set-up to the logic that is discussed in the next section, but nothing here hinges on the addition and a formalisation of the core part of DRT can be obtained by simply omitting all reference to ';'.

One of the virtues of the DRS language is that boxes are visually appealing. A disadvantage is that they take up a lot of space. This is especially annoying if one wants to talk about the box language and boxes need to enter formal expressions in the metalanguage. For this reason we shall switch to a linear notation in this and in the next section, but in section 4 , where we shall have occasion to discuss applications, we shall switch back to the easily readable box format again.

The basic ingredients of the DRS language are familiar from ordinary predicate logic; we have terms (constants or variables, the variables are also called discourse referents) and at least unary and binary predicate symbols. We use $t$ to range over terms, $P$ to range over unary predicate symbols, and $R$ to range over binary ones. With the help of these ingredients we build up conditions $(\varphi)$ and DRSs or boxes $(K)$ by the following rules, which are presented in Backus Naur Form.

$$
\begin{array}{lll}
\varphi::= & P t\left|t_{1} R t_{2}\right| t_{1}=t_{2}|\neg K| K_{1} \vee K_{2} \mid K_{1} \Rightarrow K_{2} \\
K::= & {\left[x_{1} \ldots x_{n} \mid \varphi_{1}, \ldots, \varphi_{m}\right] \mid K_{1} ; K_{2}}
\end{array}
$$

In the second clause it is to be understood that $n$ or $m$ may be equal to zero. The set of discourse referents $\left\{x_{1}, \ldots, x_{n}\right\}$ is called the universe of $\left[x_{1} \ldots x_{n} \mid \varphi_{1}, \ldots, \varphi_{m}\right]$ and the conditions $\varphi_{1}, \ldots, \varphi_{m}$ are the conditions of this DRS. As an example of a formula in this revised box language a linear alternative for (6) is given in (24) and an alternative for (19) is given in (25).

(24) $\quad[x \mid x=$ john, $\neg[\mid \neg[y \mid$ umbrella $y, x$ brought $y]]]$

(25) $[\mid[x \mid$ bathroom $x$, in-this-house $x] \Rightarrow[y \mid$ finny-place $y, y=x]]$

Next, for each condition $\varphi$ occurring in some DRS $K_{O}$ we are interested in the set $\operatorname{ACC}(\varphi)$ of discourse referents that are accessible from $\varphi$ (in $K_{O}$ ) and it will be expedient to define $\mathrm{ACC}(K)$ for each $K$ that is a subDRS of $K_{0}$ as well. Clearly no discourse referent should be accessible from $K_{0}$ itself, so we set $\operatorname{ACC}\left(K_{0}\right)=\varnothing$. Define $\operatorname{ADR}(K)$, the set of active discourse referents of any DRS $K$ by letting $\operatorname{ADR}\left(\left[x_{1} \ldots x_{n} \mid \varphi_{1}, \ldots, \varphi_{m}\right]\right)=\left\{x_{1}, \ldots, x_{n}\right\}$ and $\operatorname{ADR}\left(K_{1} ; K_{2}\right)=\operatorname{ADR}\left(K_{1}\right) \cup$ $\operatorname{ADR}\left(K_{2}\right)$. The discourse referents accessible from any subDRS or condition in $K_{O}$ can be computed in a top-down way by the following rules. 
(i) If $\mathrm{ACC}(\neg K)=X$ then $\mathrm{ACC}(K)=X$

(ii) If $\operatorname{ACC}\left(K_{1} \vee K_{2}\right)=X$ then $\operatorname{ACC}\left(K_{1}\right)=X$ and $\operatorname{ACC}\left(K_{2}\right)=X$

(iii) If $\operatorname{ACC}\left(K_{1} \Rightarrow K_{2}\right)=X$ then $\operatorname{ACC}\left(K_{1}\right)=X$ and $\operatorname{ACC}\left(K_{2}\right)=X \cup \operatorname{ADR}\left(K_{1}\right)$

(iv) If $\mathrm{ACC}\left(\left[x_{1} \ldots x_{n} \mid \varphi_{1}, \ldots, \varphi_{m}\right]\right)=X$ then $\operatorname{ACC}\left(\varphi_{i}\right)=X \cup\left\{x_{1}, \ldots, x_{n}\right\}(1 \leq i \leq m)$

(v) If $\mathrm{ACC}\left(K_{1} ; K_{2}\right)=X$ then $\operatorname{ACC}\left(K_{1}\right)=X$ and $\operatorname{ACC}\left(K_{2}\right)=X \cup \operatorname{ADR}\left(K_{1}\right)$

In order to illustrate the procedure we compute the discourse referents accessible from $y=x$ in (25): Since $\operatorname{ACC}((25))=\varnothing$ by definition, we find with rule (iv) that the set of referents accessible from (25)'s only condition is $\varnothing$; rule (iv) tells us that, since $x$ is an active discourse referent of this conditions's antecedent, ACC $([y \mid$ funny-place $y, y=x])=\varnothing \cup\{x\}=\{x\}$ and a second application of rule (iv) shows that $\mathrm{ACC}(y=x)=\{x, y\}$. If $x$ occurs in some atomic condition (i.e. condition of the form $P t$ or $t_{1} R t_{2}$ or $t_{1}=t_{2}$ ) of $K$ from which $x$ is not accessible, we say that $x$ is free in $K$. If $K$ does not contain any free discourse referents $K$ is called a proper DRS.

Our definition of the semantics of the DRT language may at first blush seem different from the one given in Kamp [1981], or Kamp \& Reyle [1993], although in fact (modulo our addition of the sequencing operator, which will have relational composition as its semantics ${ }^{10}$ ) it will be equivalent. For reasons of conciseness and easy generalisation we shall give a definition inspired by the one given in Groenendijk \& Stokhof [1991]. ${ }^{11}$ Let $M=\langle D, I\rangle$ be a first-order model with domain $D$ and interpretation function $I$ and let $f, g$ and $h$ range over finite assignments, i.e. finite partial maps from the set of discourse referents into $D$. Define $\|t\| f$ to be $f(x)$ if $t$ is the discourse referent $x$ and $x \in \operatorname{dom}(f)$, and define $\|t\| f$ to be $I(c)$ if $t$ is the constant $c$. If $f(x)$ is undef ined $\|x\| f$ will also remain undef ined. Write $f\left\{x_{1}, \ldots, x_{n}\right\} g$ if $f \subseteq g$ and $\operatorname{dom}(g)=\operatorname{dom}(f) \cup\left\{x_{1}, \ldots, x_{n}\right\}$. We define the extension $\|\varphi\|$ of a condition $\varphi$ to be a set of assignments and the extension $\|K\|$ of a DRS $K$ to be a binary relation between assignments by means of the following induction. ${ }^{12}$

DEFINITION (DRT semantics).

$\begin{array}{ll}\|P\| & =\{f \mid\|t\| f \text { is defined \& }\|t\| f \in I(P)\} \\ \left\|t_{1} R t_{2}\right\| & =\left\{f \mid\left\|t_{1}\right\| f \text { and }\left\|t_{2}\right\| \text { are defined } \&\left\langle\left\|t_{1}\right\| f,\left\|t_{2}\right\| f\right\rangle \in I(R)\right\} \\ \left\|t_{1}=t_{2}\right\| & =\left\{f \mid\left\|t_{1}\right\| f \text { and }\left\|t_{2}\right\| f \text { are defined } \&\left\|t_{1}\right\| f=\left\|t_{2}\right\| f\right\} \\ \|\neg K\| & =\{f \mid \neg \exists g\langle f, g\rangle \in\|K\|\} \\ \left\|K_{1} \vee K_{2}\right\| & =\left\{f \mid \exists g\left(\langle f, g\rangle \in\left\|K_{1}\right\| \vee\langle f, g\rangle \in\left\|K_{2}\right\|\right)\right\} \\ \left\|K_{1} \Rightarrow K_{2}\right\| & =\left\{f \mid \forall g\left(\langle f, g\rangle \in\left\|K_{1}\right\| \rightarrow \exists h\langle g, h\rangle \in\left\|K_{2}\right\|\right)\right\} \\ \left\|\left[x_{1}, \ldots, x_{n} \mid \varphi_{1}, \ldots, \varphi_{m}\right]\right\| & =\left\{\langle f, g\rangle \mid f\left\{x_{1}, \ldots, x_{n}\right\} g \& g \in\left\|\varphi_{1}\right\| \cap \ldots \cap\left\|\varphi_{m}\right\|\right\} \\ \left\|K_{1} ; K_{2}\right\| & =\left\{\langle f, g\rangle \mid \exists h\left(\langle f, h\rangle \in\left\|K_{1}\right\| \&\langle h, g\rangle \in\left\|K_{2}\right\|\right)\right\}\end{array}$

A proper DRS $K$ is true iff the empty map $\varnothing$ is an element of the domain of $\|K\|$, or, in other words, iff some $g$ with the universe of $K$ as its domain is in the extension of all conditions of $K$.

The present definition gives us the possibility to define a natural notion of equivalence in meaning: two DRSs or conditions are called equivalent iff their extensions coincide. It is easy to see that $\left[\mid \neg K_{1}\right] \vee\left[\mid K_{1} \Rightarrow K_{2}\right]$ is equivalent with $K_{1} \Rightarrow K_{2}$, and hence that Roberts' (18), discussed in the previous section, is equiva- 
lent with the simpler (19). The reader may also note that $K \vee\left[x_{1}, \ldots, x_{n} \mid \varphi_{1}, \ldots, \varphi_{m}\right]$ and $K \vee\left[x_{1}, \ldots, x_{n} \mid \neg K, \varphi_{1}, \ldots, \varphi_{m}\right]$ are equivalent. This means that the revised construction rule $A$ which we have considered in the previous section would give an output that is not semantically different from the output we get from the standard DRT construction rules. Similarly, since [ $\left.\mid \neg K_{l}\right] \Rightarrow K_{2}$ is equivalent with $K_{1} \vee K_{2}$, adopting rule $B$ would have no semantic effects either. There is a semantic difference between (6) and (7) though, and since we want a version of DRT in which double negations can be cancelled we shall define a new negation in the next section.

\section{Double Negation DRT}

The basic problem with negation in standard DRT is that it is not a flip-flop operation like its cousin in ordinary logic. Even the very syntax of negation discourages flip-flop behaviour: if $K$ is a box, $\neg K$ is a condition and there is no comparable operator which takes us from conditions to boxes again. In our variant of DRTDouble Negation DRT - we remedy this and let the negation $\sim K$ of a box $K$ itself be a box. This is our only addition and we have removed the original negation, so that the syntax of Double Negation DRT looks as follows.

$$
\begin{aligned}
& \varphi::=\quad P t\left|t_{1} R t_{2}\right| t_{1}=t_{2}\left|K_{1} \vee K_{2}\right| K_{1} \Rightarrow K_{2} \\
& K::=\left[x_{1} \ldots x_{n} \mid \varphi_{1}, \ldots, \varphi_{m}\right]\left|K_{1} ; K_{2}\right| \sim K
\end{aligned}
$$

We interpret this language by borrowing a technique from partial logic. Conditions will as before have an extension which consists of a set of partial assignments, but with each DRS $K$ two relations between assignments will be associated, its extension $\|K\|^{+}$and its anti-extension $\|K\|^{-}$. In the definition below we give the semantics of Double Negation DRT. The idea is that all conditions, except those of the form $K_{1} \vee K_{2}$, have a semantics that does not differ from the one given in the previous set-up and that the semantics of $K_{1} \vee K_{2}$ is no different from that of $\sim K_{1} \Rightarrow K_{2}$. The extension of a non-negated box $K$ is as before, but its anti-extension is defined to be equal to the extension of $[\mid \neg K]$ in the previous set-up. Negation is now indeed a flip-flop operator and switches between extensions and anti-extensions.

$$
\begin{aligned}
& \text { DEFINTTION (Double Negation DRT semantics). } \\
& \|P t\| \quad=\{f \mid\|t\| f \text { is defined \& }\|t\| f \in I(P)\} \\
& \left\|t_{1} R t_{2}\right\| \quad=\left\{f \mid\left\|t_{1}\right\| f^{f} \text { and }\left\|t_{2}\right\|^{f} \text { are defined } \&\left\langle\left\|t_{1}\right\| f,\left\|t_{2}\right\|\right\rangle \in I(R)\right\} \\
& \left\|t_{1}=t_{2}\right\| \quad=\left\{f \mid\left\|t_{1}\right\| f \text { and }\left\|t_{2}\right\| f \text { are defined } \&\left\|t_{1}\right\| f=\left\|t_{2}\right\| f\right\} \\
& \left\|K_{1} \vee K_{2}\right\| \quad=\left\{f \mid \forall g\left(\langle f, g\rangle \in\left\|K_{1}\right\|^{-} \rightarrow \exists h\langle g, h\rangle \in\left\|K_{2}\right\|^{+}\right)\right\} \\
& \left\|K_{1} \Rightarrow K_{2}\right\| \quad=\left\{f \mid \forall g\left(\langle f, g\rangle \in\left\|K_{1}\right\|^{+} \rightarrow \exists h\langle g, h\rangle \in\left\|K_{2}\right\|^{+}\right)\right\} \\
& \left\|\left[x_{1}, \ldots x_{n} \mid \varphi_{1}, \ldots, \varphi_{m}\right]\right\|^{+}=\left\{\langle f, g\rangle \mid\left\{\left\{x_{1}, \ldots, x_{n}\right\} g \& g \in\left\|\varphi_{1}\right\| \cap \ldots \cap\left\|\varphi_{m}\right\|\right\}\right. \\
& \left\|\left[x_{1}, . x_{n} \mid \varphi_{1}, \ldots, \varphi_{m}\right]\right\|^{-}=\left\{\langle f, f\rangle \mid \neg \exists g\left(\left\{\left\{x_{1}, \ldots, x_{n}\right\} g \& g \in\left\|\varphi_{1}\right\| \cap \ldots \cap\left\|\varphi_{m}\right\|\right)\right\}\right. \\
& \left\|K_{1} ; K_{2}\right\|^{+} \quad=\left\{\langle f, g\rangle \mid \exists h\left(\langle f, h\rangle \in\left\|K_{1}\right\|^{+} \&\langle h, g\rangle \in\left\|K_{2}\right\|^{+}\right)\right\} \\
& \left\|K_{1} ; K_{2}\right\|^{-} \quad=\left\{\langle f, f\rangle \mid \neg \exists g \exists h\left(\langle f, g\rangle \in\left\|K_{1}\right\|^{+} \&\langle g, h\rangle \in\left\|K_{2}\right\|^{+}\right)\right\} \\
& \|\sim K\|^{+} \quad=\|K\|^{-} \\
& \|\sim K\|^{-} \quad=\|K\|^{+}
\end{aligned}
$$


As before, two conditions are said to be equivalent iff their extensions coincide. Boxes $K_{1}$ and $K_{2}$ are equivalent iff $\left\|K_{1}\right\|^{+}=\left\|K_{2}\right\|^{+}$and $\left\|K_{1}\right\|^{-}=\left\|K_{2}\right\|^{-}$. It is immediate that $\sim \sim K$ is equivalent with $K$, whence the name 'Double Negation DRT'.

In the definition of accessibility a little care must be taken for the following reason. Clearly, in $[x \mid \operatorname{man} x] ;[y \mid$ umbrella $y, x$ owns $y]$ the first occurrence of $x$ should be accessible to the condition $x$ owns $y$. (Note that the DRS is equivalent to [ $\begin{array}{ll}x & y\end{array}$ man $x$, umbrella $y, x$ owns $\left.y\right]$.) But in $\sim[x \mid$ man $x] ;[y \mid$ umbrella $y, x$ owns $y]$ this should not be the case, while in $\sim[x \mid$ man $x] ;[y \mid$ umbrella $y, x$ owns $y]$ the accessibility should be restored again. To get this right we do not only define the set of active discourse referents of a given DRS this time, we also define its set of passive discourse referents. The following clauses do the job.

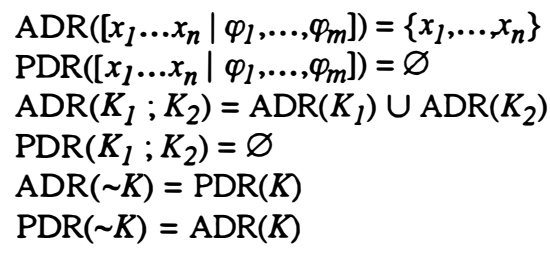

Accessibility in $K$ can now be defined in a straightforward way by setting $\operatorname{ACC}(K)=\varnothing$ and computing the accessible discourse referents of subDRSs and subconditions with the help of the following rules.

(i) If $\operatorname{ACC}\left(K_{1} \vee K_{2}\right)=X$ then $\operatorname{ACC}\left(K_{1}\right)=X$ and $\operatorname{ACC}\left(K_{2}\right)=X \cup \operatorname{PDR}\left(K_{1}\right)$

(ii) If $\operatorname{ACC}\left(K_{1} \Rightarrow K_{2}\right)=X$ then $\operatorname{ACC}\left(K_{1}\right)=X$ and $\operatorname{ACC}\left(K_{2}\right)=X \cup \operatorname{ADR}\left(K_{1}\right)$

(iii) If $\operatorname{ACC}\left(\left[x_{1} \ldots x_{n} \mid \varphi_{1}, \ldots, \varphi_{m}\right]\right)=X$ then $\operatorname{ACC}\left(\varphi_{i}\right)=X \cup\left\{x_{1}, \ldots, x_{n}\right\}(1 \leq i \leq m)$

(iv) If $\mathrm{ACC}\left(K_{1} ; K_{2}\right)=X$ then $\operatorname{ACC}\left(K_{1}\right)=X$ and $\operatorname{ACC}\left(K_{2}\right)=X \cup \operatorname{ADR}\left(K_{1}\right)$

(v) If $\operatorname{ACC}(\sim K)=X$ then $\operatorname{ACC}(K)=X$

Again, an occurrence of $x$ in an atomic condition $\varphi$ in $K$ is said to be free in $K$ iff $x \notin \mathrm{ACC}(\varphi)$. An occurrence of $x$ in an atomic condition $\varphi$ in a condition $\psi$ is free in $\psi$ iff it is free in [ $\mid \psi]$. A DRS $K$ is proper iff no occurrence of a discourse referent in $K$ is free in $K$. A proper DRS is true if $\varnothing$ is an element of the domain of its extension, false if $\varnothing$ is an element of the domain of its anti-extension. The following lemma is of practical importance.

MERGING LEMMA.

$\left[x_{1} \ldots x_{n} \mid \varphi_{1}, \ldots, \varphi_{m}\right] ;\left[y_{1} \ldots y_{k} \mid \psi_{1}, \ldots, \psi_{j}\right]$

is equivalent with

$\left[x_{1} \ldots x_{n} y_{1} \ldots y_{k} \mid \varphi_{1}, \ldots, \varphi_{m}, \psi_{1}, \ldots, \psi_{j}\right]$,

provided no referent in $y_{1}, \ldots, y_{k}$ is free in any of $\varphi_{1}, \ldots, \varphi_{m}$. 


\section{Applications}

Since in this section we want to show how our new version of DRT deals with the kind of sentences that we have encountered in the first section, we must make clear how its construction algorithm works. Fortunately we can borrow many rules from the standard approach. The basic set-up is as follows (compare the following rule for the global structure of DRS construction with that of Kamp \& Reyle $[1993$, p. 86]).

CONSTRUCTION ALGORITHM
$\begin{aligned} & \text { Input: } \quad \begin{array}{l}\text { a discourse } S_{1}, \ldots, S_{n} \\ \text { the empty DRS } K_{O}=\square\end{array} \\ & \text { For } i=1 \text { to } n \text { do: } \\ & \text { Let } K_{i}^{*}=K_{i-1} ; S_{i} \cdot 13 \text { Go to (ii). } \\ & \text { (ii) Keep on applying construction rules to each reducible condition of } \\ & K_{i}^{*} \text { until a DRS } K_{i} \text { is obtained that only contains irreducible } \\ & \text { conditions. }\end{aligned}$

Applying one step of this algorithm to (5), reprinted as (26) below, gives (27) as an output.

(26) It is not true that John didn't bring an umbrella. It was purple and it stood in the hallway.

$$
\text { It is not true that John didn 't bring an umbrella }
$$

In (27) we encounter a negation and a proper name. For these we have construction rules that are slightly different from their standard variants. They are formulated as follows.

NEGATION RULE. Upon encountering any form of linguistic negation, prefix the box that the condition containing the negation belongs to with and remove the linguistic negation.

PROPER NAME RULE. Upon encountering a proper name $\alpha$, replace $\alpha$ with a new discourse referent $x$ and prefix the entire DRS under construction with $[x \mid \alpha=x]$.

This exhausts our changes to the construction algorithm. An application of the negation rule to (27) gives (28) and a subsequent application of the proper name rule (29). In the latter we may (if we wish) merge $[x \mid$ john $=x]$ and the empty box [ $\mid]$ to $[x \mid$ john $=x]$, according to the merging lemma of the previous section. This gives (30) and with a second application of the negation rule we obtain (31). 
(28)

$\square ; ~ \sim ~ J o h n$ didn't bring an umbrella

(29)

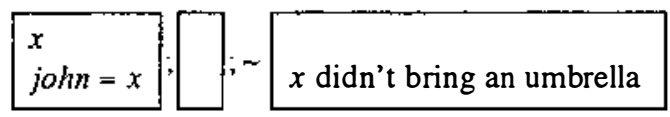

(30)

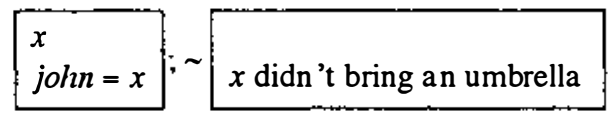

(31)

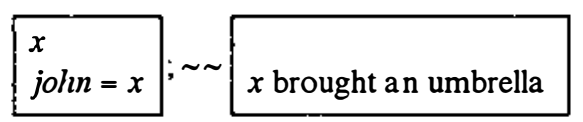

At this crucial point we may cancel the double negation, with (32) as a result, and an application of the standard rule for indefinites brings us to (33). Now the Merging Lemma can be applied, so that we get (34).

$$
\begin{array}{|l|l|}
\hline x \\
\text { jolnn }=x
\end{array} x \text { brought a n umbrella }
$$

\begin{tabular}{|l|l|}
\hline$x$ & $\begin{array}{l}y \\
\text { umbrella } y \\
x \text { brought } y\end{array}$ \\
\hline
\end{tabular}

$$
\begin{array}{|l|}
\hline x \quad y \\
\text { john }=x \\
\text { umbrella } y \\
x \text { brought } y
\end{array}
$$

Since there are no more reducible conditions now, the construction algorithm prescribes attaching a new box with the second sentence of our discourse as its only condition. The result is given in (35). Clearly, since $y$ is accessible from this new condition, both occurrences of $i t$ can be resolved as $y$.

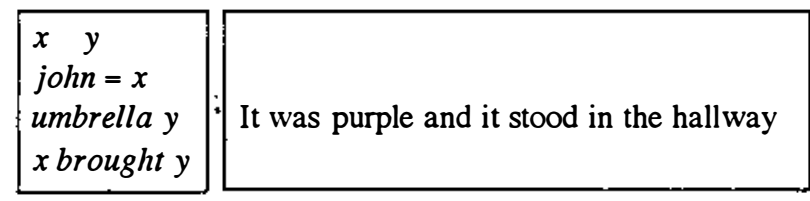

This shows that our version of DRT treats double negations as holes for anaphora. That it treats single negations as plugs can be illustrated from the treatment of (36). ${ }^{14}$ Since the only difference between the first sentence of (26) and that 
of (36) is that the latter lacks a negation, it is obvious that the construction algorithm outputs (37) instead of (33) for this sentence. This box can no further be reduced and if the second sentence of (36) is added, as in (38), we find that the two occurrences of $i t$ cannot be resolved as $y$ since the latter referent is not accessible.

(36) John didn't bring an umbrella. *It was purple and it stood in the hallway.
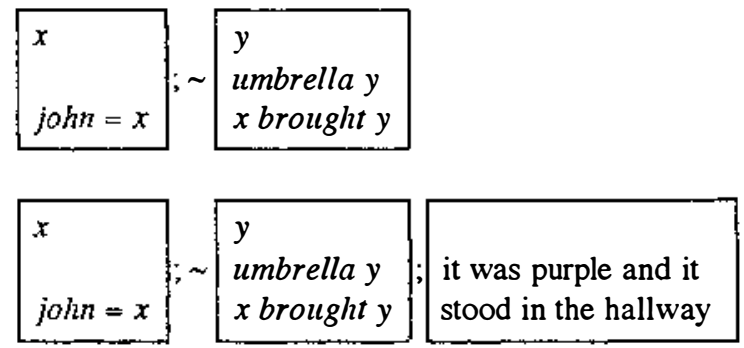

This brings us to the treatment of 'bathroom' sentences. Supposing that the construction algorithm assigns (40) to (12) (here reprinted as (39)), we see that these sentences no longer form a problem. Since $x$ is an active discourse referent of $[x \mid$ bathroom $x$, in this house $x]$, it is a passive discourse referent of its negation. This means that it will be accessible from the second disjunct, so that we can resolve it as $x$. The result is shown in (41). Note that this last box is equivalent to (19) (reprinted as (42)), so that (39) is predicted to be equivalent with (43).

(39) Either there's no bathroom in this house, or it's in a funny place

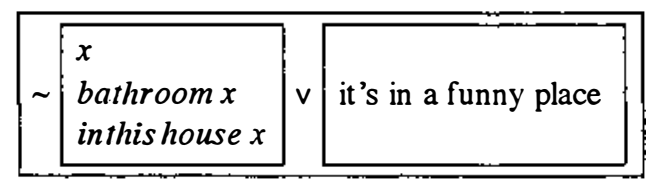

(41)
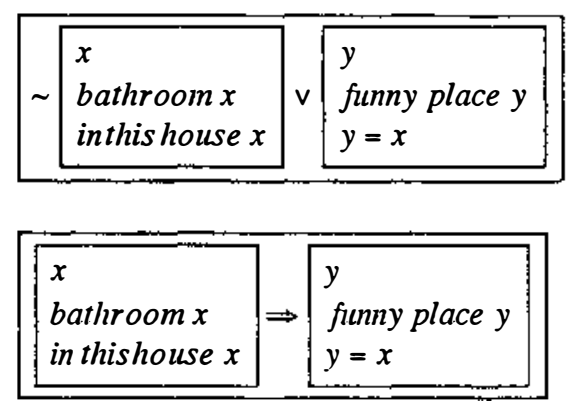

(43) If there's a bathroom in this house it's in a funny place 
A final word on representations. In this paper we have used a representation language that extends the familiar DRT language and for some discourses the DRS that we obtain after applying the construction algorithm will not be equivalent to a DRS of the old language. Thus while the DRS for the first sentence of (26) turned out to be part of the old language, the box in (37) could not be so reduced. Theoretically there is no problem here, but since discourses with an alternation of negated and non-negated sentences tend to get rather long DRSs and also for the sake of comparison with results the standard DRT set-up, we may nevertheless want to use the old forms. To this end we may reintroduce the 'old' DRT negation into the new language, stipulate that $\neg K$ is a condition if $K$ is a box, and give the following semantics.

$\|\neg K\|=\left\{f \mid \neg \exists g\langle f, g\rangle \in\|K\|^{+}\right\}$

The notion of accessibility is extended in the obvious way. We now have the following usef ul lemma which has a simple proof.

SINGLE NEGATION LEMMA

$K \Rightarrow \sim\left[x_{1} \ldots x_{n} \mid \varphi_{1}, \ldots, \varphi_{m}\right] \quad$ is equivalent with $K \Rightarrow\left[\mid \neg\left[x_{1} \ldots x_{n} \mid \varphi_{1}, \ldots, \varphi_{m}\right]\right]$

$\sim\left[x_{1} \ldots x_{n} \mid \varphi_{1}, \ldots, \varphi_{m}\right] \Rightarrow K \quad$ is equivalent with $\left[\mid \neg\left[x_{1} \ldots x_{n} \mid \varphi_{1}, \ldots, \varphi_{m}\right]\right] \Rightarrow K$

$K ; \sim\left[x_{1} \ldots x_{n} \mid \varphi_{1}, \ldots, \varphi_{m}\right] \quad$ is equivalent with $K ;\left[\mid \neg\left[x_{1} \ldots x_{n} \mid \varphi_{1}, \ldots, \varphi_{m}\right]\right]$

$\sim\left[x_{1} \ldots x_{n} \mid \varphi_{1}, \ldots, \varphi_{m}\right] ; K \quad$ is equivalent with $\left[\mid \neg\left[x_{1} \ldots x_{n} \mid \varphi_{1}, \ldots, \varphi_{m}\right]\right] ; K$

Since we can cancel double negations, since we can trade disjunctions for implications via the equi valence between $K_{1} \vee K_{2}$ and $\sim K_{1} \Rightarrow K_{2}$ and in virtue of the properties of the construction algorithm, we can now reduce our new DRSs to the old ones. The procedure is illustrated for (37) below. To this DRS the Single Negation Lemma applies, and we get (44). A last application of the Merging Lemma results in (45), the form that we are used to associate with the first sentence of (36).

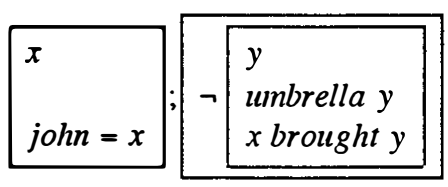

$$
\begin{aligned}
& \begin{array}{l}
x \\
\text { john }=x
\end{array} \\
& \neg \begin{array}{l}
y \\
\text { umbrella } y \\
x \text { brought } y
\end{array}
\end{aligned}
$$




\section{Endnotes}

* We would like to thank Nick Asher, David Beaver, Greg Carlson, Robin Cooper, Paul Dekker, Klaus von Heusinger, Makoto Kanazawa, William Ladusaw, Luuk Lagerwerf, Mieke Rats, Craige Roberts, Leonoor Oversteegen, Stanley Peters and Carel van Wijk for comments and criticisms.

1. Examples (1)-(4) are taken from Karttunen's original paper (Karttunen [1976]).

2. E.g. Chierchia [1992], Groenendijk \& Stokhof [1991], Kamp \& Reyle [1993].

3. We assume that negative verbs such as fail and for get should be analysed with the help of a negation (see e.g. Karttunen \& Peters [1979] for a similar analysis).

4. Roberts [1989] attributes this sentence to Barbara Partee. In Evans [1977] we find Either John does not own a donkey, or he keeps it very quiet.

5. For a discussion of the issue of accessibility in disjunctions see section 2.3.1 (pp. 185-190) of this work.

6. In fact, in a sense that will be made precise in section 2, (17) and (13) are equivalent in meaning and the new rule that in construing the DRS for a dis junction we should add the negation of the DRS for the first disjunct as a condition to the DRS for the second does not give us any output that is semantically different from the output of the original construction rule.

7 Roberts uses a modal box instead of an implication, to be quite precise, but this is immaterial for our present purposes.

8. In section 4 below we shall give a slightly different analysis of disjunctions. We shall not change the DRS construction rule for disjunctions, but the semantics for the symbol $\vee$ will be altered in such a way that $A$ or $B$ will be semantically equivalent to if not $A$ then $B$. In an earlier version of this paper our analysis of 'bathroom' sentences was based on Kamp \& Reyle's analysis plus our solution to the double negation problem. We wish to thank Paul Dekker for insisting that the equivalence between $A$ or $B$ and if not $A$ then $B$ should be retained.

9. This is the same as the conjunction operation in Groenendijk \& Stokhof [1991].

10. This is the standard semantics of sequencing - see Pratt [1976].

11. See definition 26 in Groenendijk \& Stokhof's [1991].

12. The definition that is given here is very close to Groenendijk \& Stokhof's [1991] definition 26, but we follow the standard DRT set-up by

(i) using finite (and hence partial) assignments instead of total assignments,

(ii) disallowing what are called reassignments.

The use of finite assignments has been argued for extensively by Fernando [1992]. As for (ii), note that our definition of $f\{\bar{x}\} g$ has the consequence that for example in $[x \mid$ donkey $x] \Rightarrow[x \mid$ grey $x]$ the occurrence of $x$ in the universe of the second box has no effect at all and that the condition is equivalent to $[x \mid$ donkey $x] \Rightarrow[\mid$ grey $x]$. In the Groenendijk \& Stokhof set-up it will be equivalent to $[x \mid$ donkey $x] \Rightarrow$ $[y \mid$ grey $y]$. Of course, such conditions will not be generated by the standard DRT construction algorithm. 
13. A more precise account would have the syntactic analysis of $S_{i}$ as the contents of the new box. Compare Kamp \& Reyle [1993].

14. In this respect our negation is different from the dynamic negations considered in Groenendijk \& Stokhof [1990] and Dekker [1993]. While these negations correctly predict that a double negation does not block anaphora they also predict that a single negation does not.

\section{References}

Chierchia, G.: 1992, Anaphora and Dynamic Logic, Linguistics and Philosophy 15,111-183.

Dekker, P.: 1993, Transsentential meditations - Ups and downs in Dynamic Semantics, PhD-dissertation, ILLC dissertation series, no. 1, Amsterdam.

Evans, G.: 1977, Pronouns, Quantifiers, and Relative Clauses (1 \& 2), The Canadian Journal of Philosophy 7, 467-536, 777-797.

Fernando, T.: 1992, Transition Systems and Dynamic Semantics, Logics in AI, LNCS 633, Springer-Verlag, Berlin.

Groenendijk, J. \& M. Stokhof: 1991, Dynamic Predicate Logic, Linguistics and Philosophy 14, 39-100.

Heim, I.: 1982, The Semantics of Definite and Indefinite Noun Phrases, PhD-dissertation, University of Massachusetts, Amherst.

Heim, I.: 1983, On the Projection Problem for Presuppositions, in: M. Barlow et al (eds.), Proceedings of the Second West Coast Conference on Formal Linguistics, Stanford University, Stanford, pp. 114 - 125.

Kadmon, N.: 1987, On Unique and Non-Unique Reference and Asymmetric Quantification, PhD-dissertation, University of Massachusetts, Amherst.

Kamp, H.: 1984, A Theory of Truth and Semantic Representation, in: J. Groenendijk et al (eds), Truth, Interpretation and Information, Foris Publications, Dordrecht (originally published in 1981).

Kamp, H. \& U. Reyle: 1993, From Discourse to Logic, Kluwer, Dordrecht.

Karttunen, L.: 1976, Discourse Referents, In: J. McCawley (ed.), Syntax and Semantics 7, Notes from the Linguistic Underground, Academic Press, New York, pp 363-385.

Karttunen, L \& S. Peters: 1979, Conventional Implicature, in: C. Oh \& D. Dinneen Syntax and Semantics, Vol 11: Presupposition, Academic Press, New York, pp. 1 - 56.

Pratt, V.: 1976, Semantic Considerations on Floyd-Hoare Logic, in: Proceedings of the 17th IEEE Symposium on Foundations of Computer Science, pp 109121.

Roberts, C.: 1989, Modal subordination and pronominal anaphora in discourse. Linguistics and Philosophy 12, 683-721. 